\title{
Spatial statistical analysis of Coronavirus Disease 2019 (Covid-19) in China
}

\author{
Huling Li, ${ }^{1}$ Hui Li, ${ }^{2}$ Zhongxing Ding, ${ }^{3}$ Zhibin Hu, ${ }^{3}$ Feng Chen, ${ }^{3}$ Kai Wang, ${ }^{4}$ Zhihang Peng, ${ }^{3}$ \\ Hongbing Shen $^{3}$ \\ ${ }^{1}$ College of Public Health, Xinjiang Medical University, Urumqi; ${ }^{2}$ Central Laboratory of Xinjiang Medical \\ University, Urumqi; ${ }^{3}$ Department of Epidemiology and Biostatistics, School of Public Health, Nanjing \\ Medical University, Nanjing, Jiangsu; ${ }^{4}$ Department of Medical Engineering and Technology, Xinjiang
}

\begin{abstract}
The cluster of pneumonia cases linked to coronavirus disease 2019 (Covid-19), first reported in China in late December 2019 raised global concern, particularly as the cumulative number of cases reported between 10 January and 5 March 2020 reached 80,711 . In order to better understand the spread of this new virus, we characterized the spatial patterns of Covid-19 cumulative cases using ArcGIS v.10.4.1 based on spatial autocorrelation and cluster analysis using Global Moran's I (Moran, 1950), Local Moran's I and Getis-Ord General G (Ord and Getis, 2001). Up to 5 March 2020, Hubei Province, the origin of the Covid-19 epidemic, had reported 67,592 Covid-19 cases, while the confirmed cases in the surrounding provinces Guangdong, Henan, Zhejiang and Hunan were 1351, 1272, 1215 and 1018, respectively. The top five
\end{abstract}

Correspondence: Hongbing Shen, Nanjing Medical University, Nanjing, 211166 Jiangsu, China.

E-mail: hbshen@njmu.edu.cn

Key words: Coronavirus disease 2019 (Covid-19); Hubei province area; Spatial statistics; Clusters; China.

Contributions: All authors conceived the study, carried out the analysis, discussed the results, drafted the first manuscript, critically read and revised the manuscript, and gave final approval for publication. All authors have read and agreed to the published version of the manuscript.

Funding: This work was supported in part by the Natural Science Foundation of China $(11961071,81673275)$ and the National S $\backslash \& T$ Major Project Foundation of China (2018ZX10715002-004-002, 2018ZX10713001-001).

Conflicts of Interest: The authors declare no conflict of interest.

Received for publication: 23 February 2020

Accepted for publication: 22 March 2020.

CCopyright: the Author(s), 2020

Licensee PAGEPress, Italy

Geospatial Health 2020; 15:867

doi:10.4081/gh.2020.867

This article is distributed under the terms of the Creative Commons Attribution Noncommercial License (CC BY-NC 4.0) which permits any noncommercial use, distribution, and reproduction in any medium, provided the original author(s) and source are credited. regions with respect to incidence were the following provinces: Hubei (11.423/10,000), Zhejiang (0.212/10,000), Jiangxi $(0.201 / 10,000)$, Beijing $(0.196 / 10,000)$ and Chongqing $(0.186 / 10,000)$. Global Moran's $I$ analysis results showed that the incidence of Covid-19 is not negatively correlated in space $(p=0.407413>0.05)$ and the High-Low cluster analysis demonstrated that there were no high-value incidence clusters $(p=0.076098>0.05)$, while Local Moran's $I$ analysis indicated that Hubei is the only province with High-Low aggregation $(p<0.0001)$.

\section{Introduction}

Two different viruses, the Severe Acute Respiratory Syndrome Coronaviruses (SARS-CoV) and the Middle East Respiratory Syndrome Coronaviruses (MERS-CoV), have caused human infections of alarming morbidity and mortality (Paules et al., 2020). These epidemics occurred in the early 21 th century, the first in China and the latter in Saudi Arabia, were contained relatively soon after detection. Further research has discovered six new such strains of virus with capacity to infect humans ( $\mathrm{HCoV})$ : two $\alpha \mathrm{CoV}$ (HCoV-229E and HKU-NL63) and four $\beta \mathrm{CoV}$ (HCoVOC43 lineage A, HCoV-HKU1 lineage A, SARS-CoV lineage B and MERS-CoV lineage C), (Chan et al., 2020a). However, no case of $\mathrm{HCoV}$ was reported until Wuhan, the capital of Hubei Province in China, was hit in late November 2019 by SARS-CoV2 , the cause of coronavirus disease 2019 (Covid-19) (Zhu et al., 2020). Within just a few weeks, Covid-19 pneumonia spilled into all other provinces and other administrative areas of China resulting in strict efforts by the Chinese Government to contain its spread (Wang et al., 2020). From late January 2020 cases were discovered also in neighbouring countries, and when the numbers in these areas grew above 50 already the following month, the world faced a global emergency. In early March, the World Health Organization (WHO) confirmed that many countries, such as the Republic of Korea $(6,284)$, Italy $(3,858)$, Iran $(3,513)$, Germany (534), France (420), Japan (349), Spain (257), USA (148) and the United Kingdom (118) had noted rising numbers of cases (WHO, 2020). Almost everywhere in the world, first cases were soon reported, e.g., in USA (Holshue et al., 2020).

Already in the early phase of the outbreak (from 27 November 2019 to 22 January 2020), Li et al. (2020) reported the presence of person-to-person transmission. Based on demographic characteristics of 425 patients with confirmed infections, exposure history and timelines of illness, they were able to analyse the early transmission dynamics of the then novel Covid-19 pneumonia in Wuhan, finding in this period of exponential growth a mean incu- 
bation period of 5.2 days ( $95 \%$ CI: 4.1 to 7.0 ). With a mean serial interval of 7.5 days ( $95 \%$ CI: 5.3 to 19$)$, the basic reproductive number $\left(\mathrm{R}_{0}\right)$ was estimated to be $2.2(95 \% \mathrm{CI}$ : 1.4 to 3.9$)$ ( $\mathrm{Li}$ et al., 2020). In another recent study of a familial cluster of pneumonia caused by SARS-CoV-2, Chan et al. (2020b) commented on the possibility of intercity spread of the new virus by air travel, thus supporting reports of infected Chinese travellers from Wuhan being detected in other geographical regions of the country. These results were corroborated by Lai et al. (2020) who reported a mean incubation period of 6.4 days and a $\mathrm{R}_{0}$ varying between 2.2 and 3.6 Another, data-driven analysis found $\mathrm{R}_{0}$ ranging from $2.2(95 \% \mathrm{CI}$ : $1.96-2.55)$ to 3.6 (95\%CI: $2.89-4.39)$ during in the early outbreak data (Zhao et al., 2020). Meanwhile, Nishiura (2020) used a spatial back-calculation method for real-time analysis of 13 exported cases to estimate the cumulative incidence of Covid-19 cases in China suggesting it to be 5,502 cases (95\% CI: 3027 to 9057 ) as of 24 January 2020, a number that was in fact not reached until 27 January.

Based on the numbers available, we demonstrate in this paper spatial cluster patterns of Covid-19 inside China, information that should prove useful for improved, future control of the epidemic.

\section{Materials and Methods}

The study was conducted in two steps: we first collected available data with respect to all confirmed Covid-19 cases in China; second, the spatial patterns of these cases were analysed by a Geographical Information Systems (GIS) approach applying ArcGIS software, version 10.4.1 (ESRI, Redlands, CA, USA) to create a spatial database, after which we applied the ArcGIS geographic statistical analysis module to investigate the spatial distribution trends.

\section{Data acquisition}

Data related to all laboratory-confirmed Covid-19 cases in the 34 administrative regions in mainland China (consisting of 23 provinces, 5 autonomous regions, 4 municipalities and 2 special administrative areas), including the total population in each region, were obtained from the National Health Commission (2020). We analysed the cumulative number of all reported cases between 10 January and 5 March 2020 as announced by WHO on March 6, 2020 (WHO, 2020) calculating the incidence of Covid-19 in each region according to the following formula:

Covid-19 incidence $=$ confirmed cases $/$ total population

\section{Statistical analysis}

Spatial autocorrelation was studied by Global Moran's I (Moran, 1950) and Local Moran's I according to Anselin (1995), while Getis-Ord General G (Ord and Getis, 2001) was used for cluster analysis. A $p$-value $<0.05$ was considered statistically significant.

\section{Global spatial trend analysis}

In general, trend analysis is to deal with points in geographical areas (here the incidence of disease) above a plot of the study site (here the whole country) calculating the attribute values (here the incidence in each unit of area) thereby creating a three-dimensional plot that allows observation of the global changes of the dataset from different perspectives. The X-axis points East, the Y-axis points North and the Z-axis refers to the number of cases in each unit of area. The points are then projected in two directions onto planes perpendicular to the geographical area, one oriented in the north-south direction and the other in the east-west direction. A polynomial curve is finally made to fit each of the two projections, which shows the trend by its curvature (a flat curve indicates that no trend exists).

\section{Spatial clustering analysis}

Spatial autocorrelation was applied to assess the spatial correlation between variables through matching location similarity and attribute similarity (Huo et al., 2012). Global Moran's $I$, an index of spatial autocorrelation based on cross products is mathematically expressed as follows:

$$
I=\frac{\sum_{i=1}^{\mathrm{n}} \sum_{j=1}^{n} w_{i j}\left(x_{i}-\bar{x}\right)\left(x_{j}-\bar{x}\right)}{\sum_{i=1}^{\mathrm{n}}\left(x_{i}-\bar{x}\right)}
$$

where $n$ is the number of regions; $x_{i}$ the attribute value at area $I ; \bar{x}$ the mean value of the attribute in the study region; and $w_{i j}$ elements of a spatial lag operator $W$ (spatial weights of matrix $W$ ). The significance of the index is usually tested in a situation of normal distribution (Mazzulla et al., 2012). Global Moran's I varies between -1 and 1 with a positive value meaning that a point in question is prone to be clustered by adjacent points, while a negative value means the opposite. Values close to 0 indicate that the data are randomly distributed.

Local Moran's I analysis (Anselin, 1995) was applied to identify statistically significant hotspots, coldspots and outliers. Local Moran's $I$ is expressed by the following formula:

$$
I_{\mathrm{i}}=x_{i} \sum_{j} w_{i j} x_{j}
$$

The variables and calculation of the Local Moran's $I$ are thus similar to those of Global Moran's $I$. It is possible to acquire the mean and the variance of $I_{i}$ based on randomization, and inference can be carried out by normalized statistic with Z-score and $p$-value indicating statistical significance. The different classes of $z$-values are represented as High-High (HH) or Low-Low (LL). High, positive $\mathrm{z}$-values indicate that an area is surrounded by areas have similar values. Conversely, a low negative $\mathrm{z}$-score indicates a statistically significant spatial anomaly, i.e. a high-value area surrounded by low-value areas (HL) with a LH area representing the inverse (Huo et al., 2011; Samphutthanon et al., 2013).

\section{Getis-Ord General G}

The $G_{i}(d)$ statistics (Ord and Getis, 2001) is a distance-based tool to measure the proportion of a variable within a given radius of a point to that in the whole study region. The statistic for loca- 
tion $i$ is defined as:

$$
G_{i}(d)=\frac{\sum_{j=1}^{n} w_{i j}(d) x_{j}}{\sum_{i=1}^{\mathrm{n}} x_{i}}
$$

where $x_{j}$ is the value of the observation at point $j ; w_{i j}(d)$ the $i j$ element of a binary $W$ matrix $\left(w_{i j}=1\right.$ if the site is within distance $d$ or 0 if elsewhere; and $n$ the number of observations made. The mean and the variance of this statistic can be obtained through randomization and used to derive a standard statistic. When the value of the standardized statistic is greater than the cut-off value with pre- specified significance, positive or negative spatial association exists. Positive values represent spatial agglomeration, while negative values represent the opposite (Páez and Scott, 2004). The higher or lower the z-score, the higher the possibility of clustering, while a z-score close to zero means absence of obvious clusters. Thus, a positive $\mathrm{z}$ represents the possibility of clustering, while a negative $\mathrm{z}$ indicates a low possibility of clustering (Weeberb et al., 2015).

\section{Results}

\section{General epidemic characteristics of Covid-19}

The incidence of Covid-19 in the 34 administrative regions provinces and cities in China (Table 1). As can be seen from

Table 1. Incidence of Covid-19 in all Chinese administrative regions.

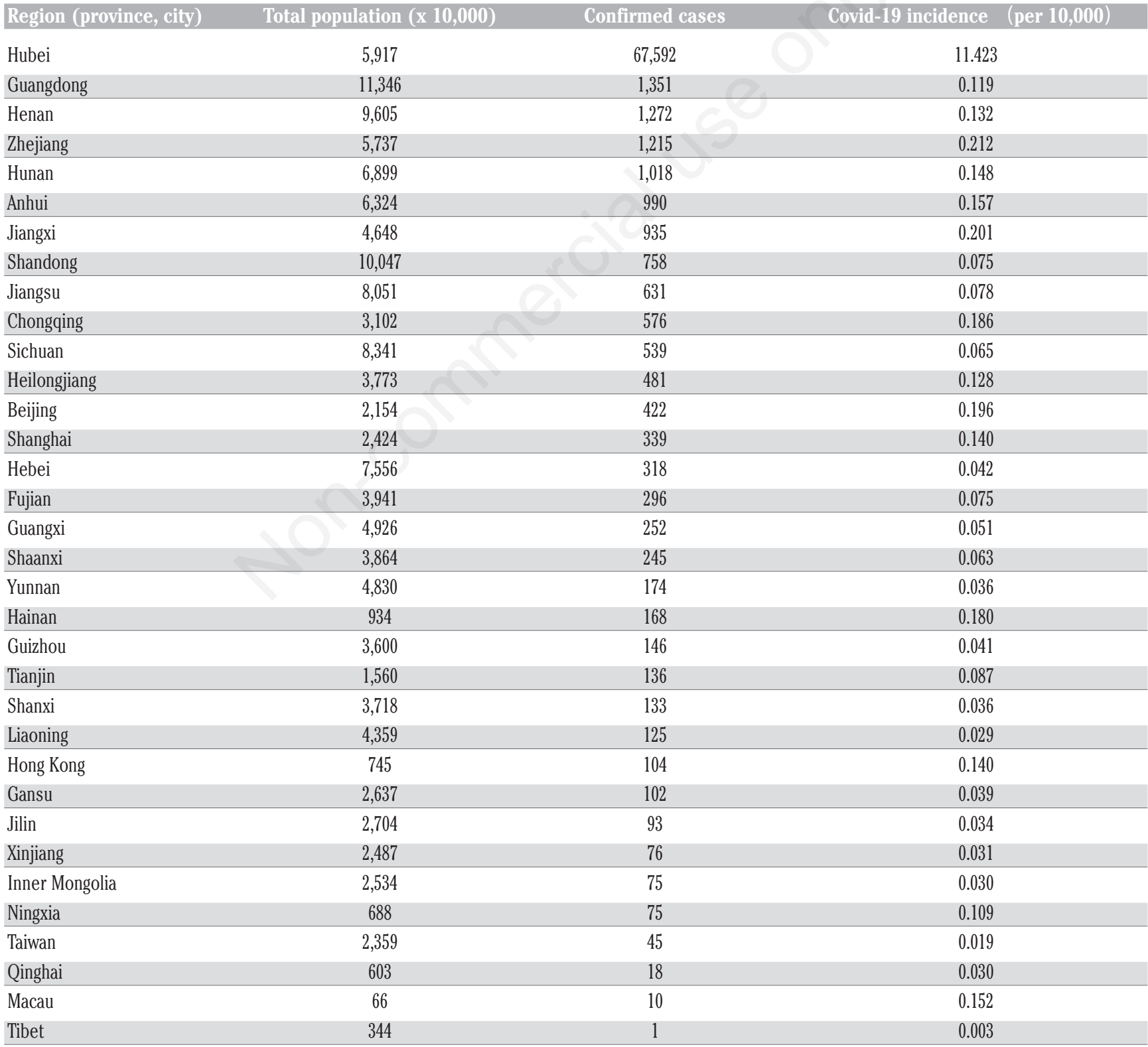

*Confirmed cases As of March 5, the incidence of Covid-19 was the number of confirmed cases per 10,000 people. 
Table 1 and Figure 1, depicting the situation as of 5 March 2020, the top five regions with respect to the number of cases of Covid19 were the following provinces: Hubei (67,592), Guangdong $(1,351)$, Henan $(1,272)$, Zhejiang $(1,215)$ and Hunan $(1,018)$. Moreover, the regions with the highest incidence were Hubei (11.423/10,000), Zhejiang (0.212/10,000), Jiangxi (0.201/10,000), Beijing $(0.196 / 10,000)$ and Chongqing $(0.186 / 10,000)$ and the central and south-eastern part of the country.

\section{Global spatial trend analysis}

The green curve on the perpendicular plane represents the trend in the east-west direction, and the blue curve on the other perpendicular plane represents the trend in the north-south direction. As shown in Figure 2, the Covid-19 incidence demonstrates a clear upward trend from west to east, while in the north-south direction the high incidence areas are mainly in the central coastal areas.

\section{Analyses of spatial patterns}

\section{Global spatial cluster analysis}

The spatial correlations of Covid-19 epidemics between the Chinese 34 administrative regions were lower than zero (negative) for Covid-19 according to Global Moran's $I$ calculations (Figure 3 ). Moran's $I$ and the $\mathrm{z}$-score were $-0.056440(p=0.407413>0.05)$, -0.828456 , respectively, indicating that the incidence of Covid-19 was not negatively and spatially correlated between regions.

\section{Getis-Ord General G}

As shown in Figure 4, the results of the HL cluster analysis showed that we did not identify high or low incidence clusters for the Covid-19 (General $\mathrm{G}<0.0001, \mathrm{z}=1.773787, p=0.076098>0.05$ ).

\section{Local spatial cluster analysis}

The results of Local Moran's $I$ analysis reveals that the value of only one location belonging to the HL aggregation class is statistically significant $(\mathrm{I}=-2.87565 \mathrm{E}-05, \mathrm{z}=-5.0559, \mathrm{p}<0.0001)$. That is, the incidence of Covid-19 in Hubei Province shows a HL aggregation type, but much less in adjacent provinces and cities. The Covid-19 incidence in Hubei Province is thus a spatial outlier. It also shows that the current COVID-19 epidemic has so far neither developed hotspots (HH) nor coldspots (LL). The results are shown in Figure 5 and Table 2.

\section{Discussion}

The emergence of SARS-CoV-2, and the disease Covid-19 that it causes, in China at the end of 2019 caused a large, local outbreak followed by a global transmission of the virus within two month due to rapid spread similar to influenza by human-to-human transmission via airborne droplets and direct contact. During the development of epidemic into pandemic, researchers have analysed the epidemiological status of Covid-19 by different methods and perspectives including mathematical models to estimate incubation times and $\mathrm{R}_{0}$. The former was found to vary between 5.2 and 6.4 (Li et al., 2020; Lai et al., 2020), while most agreed on a $\mathrm{R}_{0}$ value of 2.2 (Li et al., 2020; Lai et al., 2020; Zhao et al., 2020), although the two latter research groups reported variations reaching 3.6 during the early phase of the epidemic in China, which is of concern as these values are higher than all known previous influenza pandemics.
The combination of spatial epidemiology and GIS has been widely used in the field for the study of acute infectious diseases (Liu et al., 2013) as well as for prevention and control of chronic infections (Sun et al., 2018). At present, the spatial distribution of the reported incidence of Covid-19 in different regions across the country has not been studied in great detail.

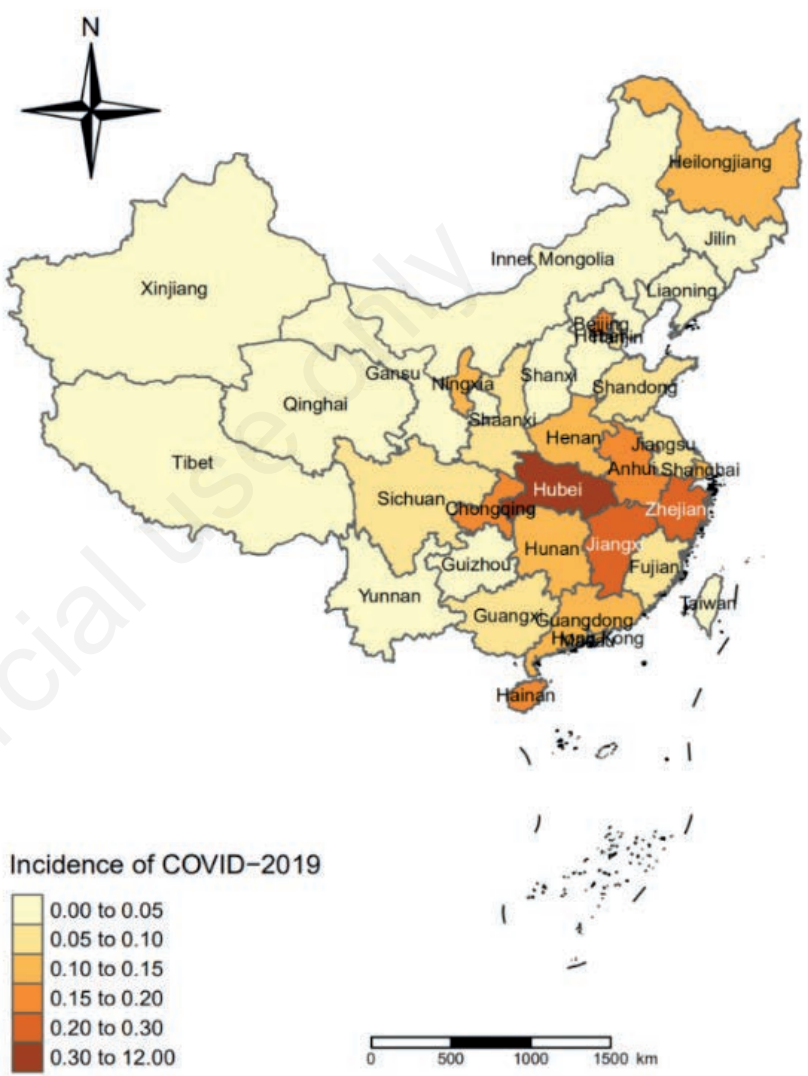

Figure 1. Geographical distribution of Covid-19 incidence.

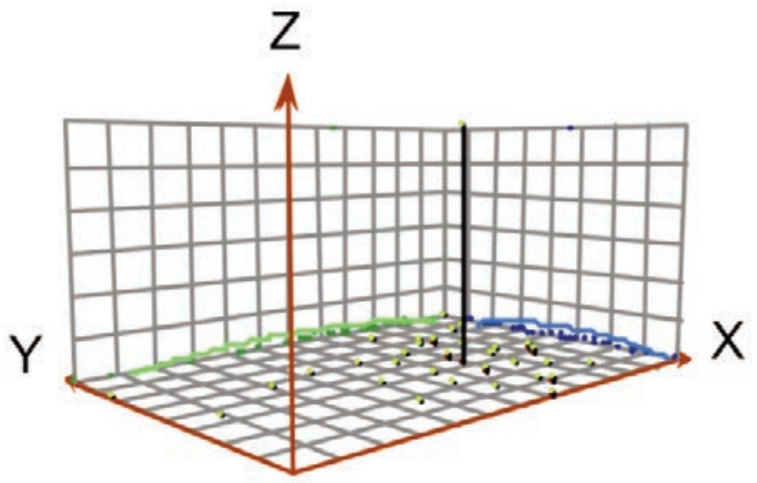

$\mathrm{Y}=$ North direction; $\mathrm{X}=$ East direction; $\mathrm{Z}=$ attribute values

Figure 2. Covid-19 incidence trend. 


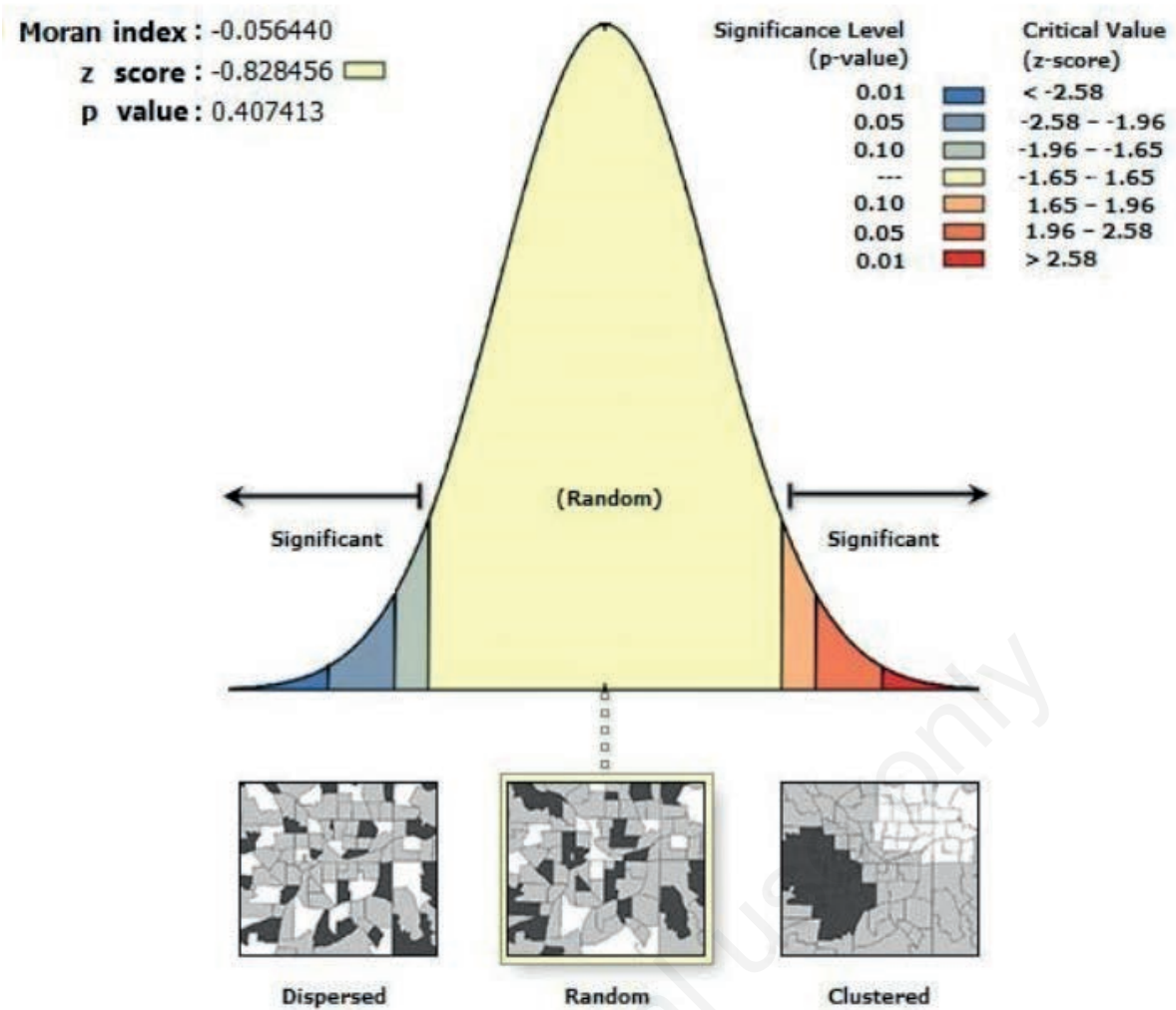

Figure 3. Global spatial autocorrelation analysis of Covid-19 incidence.

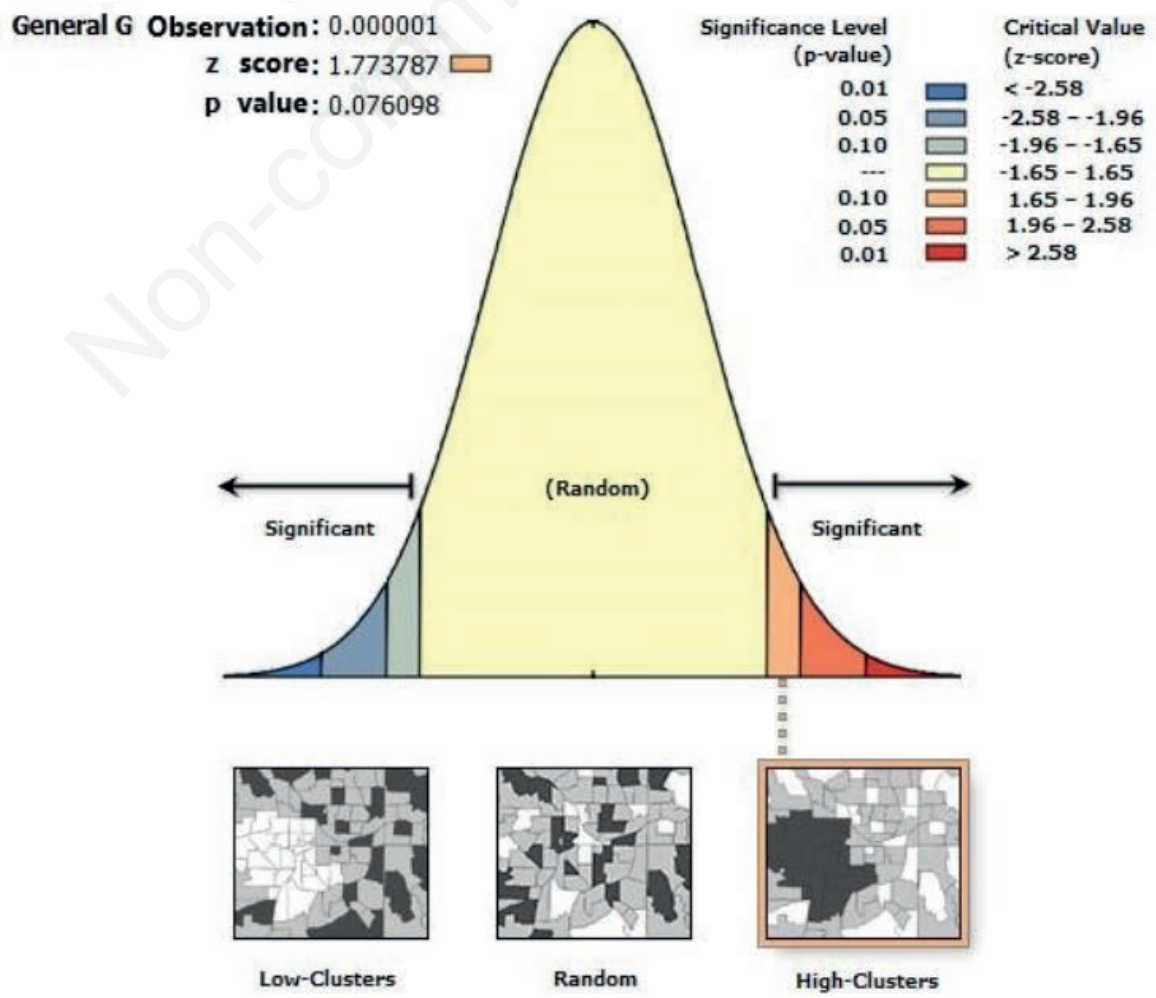

Figure 4. High-low cluster analysis of Covid-19 incidence. 
This study analysed the reported Covid-19 incidence by spatial statistical methods, exploring the geographical distribution of the disease. Using global spatial correlation analysis, we neither found any negative correlation in the spatial distribution of Covid-19 incidence nor were there any high-value clusters. While local spatial correlation analysis showed Hubei Province harbouring most of the cases and also the highest incidence, the pattern of HL aggregation made the province a spatial outlier. We also noted the lack of hotspots (HH) as well as coldspots (LL) at the end of the time covered (5 March). Due to the short period of time covered, the advantages of HL cluster analysis cannot tell us more now. Answers to the questions whether the disease will stay in the same geographic location or spread to nearby areas, and whether the measures applied to contain further spread are effective, require HL cluster analysis extended over a longer time. However, the lack of Covid-19 clusters with high or low values is an indication that in China, the focus area for Covid-19 prevention and control is still in Wuhan, Hubei Province. To prevent further spread, the Chinese Government has rapidly instituted measures to suppress transmis-

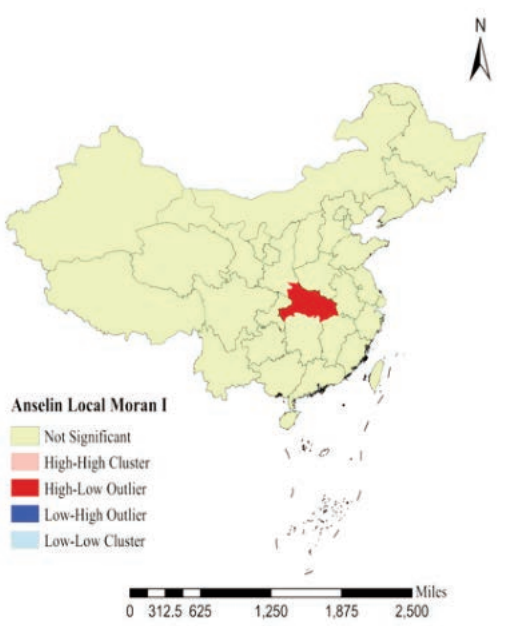

Figure 5. Local spatial autocorrelation analysis of COVID-19 incidence.

Table 2. Local spatial autocorrelation analysis of Covid-19 incidence in all Chinese regions.

\begin{tabular}{|c|c|c|c|c|c|}
\hline Region (province, city) & Incidence (per 10,000 people) & Local Moran's I & $Z$ value & $P$ value & Aggre-gation type* \\
\hline Zhejiang & 0.2118 & $-4.27918 \mathrm{E}-07$ & 0.0658 & 0.9475 & / \\
\hline Yunnan & 0.0360 & $2.59348 \mathrm{E}-07$ & 0.3335 & 0.7388 & / \\
\hline Xinjiang & 0.0306 & 4.00474E-08 & 0.2497 & 0.8028 & / \\
\hline Hong Kong & 0.1396 & $-6.07985 \mathrm{E}-08$ & 0.1447 & 0.8849 & / \\
\hline Tibet & 0.0029 & $9.25697 \mathrm{E}-08$ & 0.3099 & 0.7567 & / \\
\hline Taiwan & 0.0191 & 4.25909E-07 & 0.3384 & 0.7351 & / \\
\hline Sichuan & 0.0646 & $-7.17327 \mathrm{E}-07$ & -0.1103 & 0.9122 & / \\
\hline Shaanxi & 0.0634 & $-1.15329 \mathrm{E}-06$ & -0.0949 & 0.9244 & I \\
\hline Shanxi & 0.0358 & $-7.67979 \mathrm{E}-07$ & -0.0061 & 0.9952 & l \\
\hline Shandong & 0.0754 & $-5.00871 \mathrm{E}-07$ & 0.0664 & 0.9471 & I \\
\hline Qinghai & 0.0299 & 2.14309E-07 & 0.3371 & 0.7360 & / \\
\hline Ningxia & 0.109 & $-5.32642 \mathrm{E}-07$ & 0.0045 & 0.9964 & l \\
\hline Inner Mongolia & 0.0296 & 4.43666E-07 & 0.3641 & 0.7158 & / \\
\hline Liaoning & 0.0287 & 5.15735E-07 & 0.3656 & 0.7146 & I \\
\hline Jiangxi & 0.2012 & -8.13777E-07 & 0.0114 & 0.9909 & / \\
\hline Jilin & 0.0344 & $3.52553 \mathrm{E}-07$ & 0.3468 & 0.7288 & I \\
\hline Hunan & 0.1476 & $-1.52153 \mathrm{E}-06$ & -0.1179 & 0.9061 & / \\
\hline Hubei & 11.4234 & $-2.87565 \mathrm{E}-05$ & -5.0559 & $<0.0001$ & HL \\
\hline Heilongjiang & 0.1275 & 1.01755E-07 & 0.2513 & 0.8016 & / \\
\hline Henan & 0.1324 & $-1.8283 \mathrm{E}-06$ & -0.1911 & 0.8484 & I \\
\hline Beijing & 0.1959 & 2.11934E-07 & 0.1855 & 0.8528 & I \\
\hline Tianjin & 0.0872 & 7.04218E-08 & 0.1728 & 0.8628 & I \\
\hline Hainan & 0.1799 & 2.18074E-07 & 0.2730 & 0.7849 & / \\
\hline Guizhou & 0.0406 & $-1.01884 \mathrm{E}-06$ & -0.1150 & 0.9084 & I \\
\hline Guangxi & 0.0512 & $-7.10183 \mathrm{E}-07$ & -0.0348 & 0.9722 & I \\
\hline Gansu & 0.0387 & 3.08989E-07 & 0.3416 & 0.7327 & I \\
\hline Fujian & 0.0751 & -7.07948E-07 & 0.0043 & 0.9966 & I \\
\hline Macau & 0.1515 & $-4.72141 \mathrm{E}-08$ & 0.1454 & 0.8844 & I \\
\hline Anhui & 0.1565 & -1.01079E-06 & -0.0172 & 0.9863 & / \\
\hline Shanghai & 0.1399 & $-4.73568 \mathrm{E}-07$ & 0.0464 & 0.9630 & I \\
\hline Chongqing & 0.1857 & $-1.14389 \mathrm{E}-06$ & -0.0955 & 0.9239 & / \\
\hline Jiangsu & 0.0784 & $-6.8176 \mathrm{E}-07$ & 0.0317 & 0.9747 & I \\
\hline Guangdong & 0.1191 & $-3.31323 \mathrm{E}-07$ & 0.1063 & 0.9153 & I \\
\hline Hebei & 0.0421 & 1.37649E-07 & 0.1749 & 0.8611 & I \\
\hline
\end{tabular}

*Indicates that the correlation of between remaining areas is not significant; HL indicates a high-low aggregation type. 
sion of SARS-CoV-2 among local residents. The Chinese Spring Festival holidays were extended and school openings postponed (Yang et al., 2020), and as no specific antiviral treatment for Covid-19 is available (Wang and Zhang, 2020), self-protection is widely encouraged, such as washing hands carefully and staying home. The authorities further and initiated serious isolation measures, first for Wuhan and soon after for the whole of Hubei Province. Epidemic information, monitoring and reporting were instituted with emphasis on detection of new cases, isolation and patient care. After that, supplies were organized and measures taken to control the disease guaranteeing the order of daily life.

In response to the Covid-19 epidemic, future studies must focus on containment and, particularly, vaccine and drug development. For example, one case of SARS-CoV-2 pneumonia in USA has responded well to the drug Remdesivir, which is now undergoing a clinical trial in China (Lai et al., 2020). Such studies would help further reduction of case fatalities and transmission rates among patients infected by SARS-CoV-2. With the progress of diagnostic technology, potential patients playing the role of high-risk infectious sources might be discovered (Sun et al., 2020). Asymptomatic infections might indeed be the highest risk, something that must be investigated to achieve prevention of further spread of SARS-CoV-2 (Rothe et al., 2020). However, many other questions about this epidemic remain unanswered, pertaining to virus hosts, transmission routes, incubation periods, and susceptible populations and survival rates. In addition, viral infections can increase the risk of pulmonary fibrosis. Therefore, pulmonary fibrosis may be one of the severe complications after patients recover from this infection. The prevention of pulmonary fibrosis in patients recovered is an issue which urgently needs to be addressed (Sheng et al., 2019). Unlike SARS and MERS, where initial chest imaging abnormalities are more frequently unilateral, Covid-19 is more likely to involve both lungs (Hosseiny et al., 2020) and this evidence suggests that initial chest imaging would show would be useful for most patients. Recent studies indicate that patients aged $\geq 60$ years are at a higher risk than children, who might be less susceptible to Covid-19 infection and/or show mild or no symptoms when infected (Li et al., 2020). On 13 February, the fatality rate of all Covid-19 infections was calculated as 2.2\% $(1,370 / 60,363)$ (Velavan and Meyer, 2020). By 5 March 2020, mainland China had 80,711 accumulated Covid-19 cases, 3,045 deaths and 482 suspected cases, including as many as 53,726 people who had recovered from the infection (National Health Commission, 2020) out of which the latter must be seen as a very positive development.

\section{Conclusions}

This study identified the provinces with high and low Covid19 clusters in China. Spatial correlations between regions may provide instructive information for the control of Covid-19 epidemic in China. Follow-up HL cluster analysis with a longer time perspective is advised.

\section{References}

Anselin L, 1995. Local Indicators of Spatial Association-LISA. Geogr Anal 27:93-115. doi: 10.1111/j.1538-4632.1995 .tb00338.x.
Chan JF, Kok KH, Zhu Z, et al., 2020a. Genomic characterization of the 2019 novel human-pathogenic coronavirus isolated from a patient with atypical pneumonia after visiting Wuhan. Emerg Microbes Infect 9:221-36. doi:10.1080/22221751.2020.1719902.

Chan JF, Yuan S, Kok KH, et al., 2020b. A familial cluster of pneumonia associated with the 2019 novel coronavirus indicating person-to-person transmission: a study of a family. Lancet cluster [published online ahead of print Jan 24]. doi:10.1016/ S0140-6736(20)30154-9.

Holshue ML, DeBolt C, Lindquist S, Lofy KH, Wiesman J, et al., 2020. First Case of 2019 Novel Coronavirus in the United States. N Engl J Med 382:929-36. doi:10.1056/NEJM oa2001191.

Hosseiny M, Kooraki S, Gholamrezanezhad A, Reddy S, Myers L, 2020. Radiology Perspective of Coronavirus Disease 2019 (Covid-19): Lessons from Severe Acute Respiratory Syndrome and Middle East Respiratory Syndrome. AJR Am J Roentgenol 214:1078-82. doi: 10.2214/AJR.20.22969.

Huo XN, Li H, Sun DF, Zhou LD, Li BG, 2012. Combining geostatistics with Moran's I analysis for mapping soil heavy metals in Beijing, China. Int J Environ Res Public Health 9:9951017. doi:10.3390/ijerph9030995.

Huo XN, Zhang WW, Sun DF, Li H, Zhou LD, Li BG, 2011. Spatial pattern analysis of heavy metals in Beijing agricultural soils based on spatial autocorrelation statistics. Int J Environ Res Public Health 8:2074-89. doi:10.3390/ijerph8062074.

Li Q, Guan X, Wu P, Wang X, Zhou L, et al., 2020. Early Transmission Dynamics in Wuhan, China, of Novel Coronavirus-Infected Pneumonia. N Engl J Med 382:1199207. doi:10.1056/NEJMoa2001316.

Lai CC, Shih TP, Ko WC, Tang HJ, Hsueh PR, 2020. Severe acute respiratory syndrome coronavirus 2 (SARS-CoV-2) and coronavirus disease-2019 (Covid-19): The epidemic and the challenges [published online ahead of print]. Int $\mathrm{J}$ Antimicrob Agents 55:105924. doi:10.1016/j.ijantimicag.2020.105924

Liu W, Yang K, Qi X, Xu K, Ji H, et al. 2013. Spatial and temporal analysis of human infection with avian influenza A(H7N9) virus in China. Euro Surveill 18:20640. doi:10.2807/15607917.es2013.18.47.20640

Mazzulla G, Forciniti C, 2012. Spatial association techniques for analysing trip distribution in an urban area. Eur Transp Res Rev 4:217-33. doi:10.1007/s12544-012-0082-9.

Moran PAP, 1950. Notes on Continuous Stochastic Phenomena. Biometrika 371:17-23. doi:10.2307/2332142. JSTOR 2332142.

Nishiura H, Jung SM, Linton NM, Kinoshita R, Yang Y, Hayashi K, Kobayashi T, Yuan B, Akhmetzhanov AR, 2020. The Extent of Transmission of Novel Coronavirus in Wuhan, China, 2020. J Clin Med 9:330. doi:10.3390/jcm9020330.

Ord K, Getis A, 2001. Testing for Local Spatial Autocorrelation in the Presence of Global Spatial Autocorrelation. J Reg Sci 41:411-32. doi:10.1111/0022-4146.00224.

Páez A, Scott DM, 2004. Spatial statistics for urban analysis: A review of techniques with examples. GeoJournal 61(1), 53-67. doi:10.1007/s10708-005-0877-5.

Paules CI, Marston HD, Fauci AS, 2020. Coronavirus InfectionsMore Than Just the Common Cold. JAMA 323:707-8. doi:10.1001/jama.2020.0757.

Rothe C, Schunk M, Sothmann P, Bretzel G, Froeschl G, et al., 2020. Transmission of 2019-nCoV infection from an asymptomatic contact in Germany. N Engl J Med 382:970-1. doi:10. 1056/NEJMc 2001468 
Samphutthanon R, Tripathi N, Ninsawat S, Duboz R, 2013. Spatiotemporal distribution and hotspots of hand, foot and mouth disease (HFMD) in northern Thailand. Int J Environ Res Public Health 11:312-36.

Sheng G, Chen P, Wei Y, Yue H, Chu J, Zhao J, Wang Y, Zhang W, Zhang HL, 2019. Viral infection increases the risk of idiopathic pulmonary fibrosis: a meta-analysis. Chest doi: $10.1016 / \mathrm{j}$. chest.2019.10.032

Sun SH, Gao ZD, Zhao F, et al., 2018. [Spatial-temporal Analysis on Pulmonary Tuberculosis in Beijing During 2005-2015.] Zhonghua Liu Xing Bing Xue Za Zhi 39:816-20. [Article in Chinese]. doi:10.3760/cma.j.issn.0254-6450.2018.06.023

Sun P, Lu X, Xu C, Sun W, Pan B, 2020. Understanding of Covid19 based on current evidence [published online ahead of print, 2020 Feb 25]. J Med Virol doi:10.1002/jmv.25722.

Velavan TP, Meyer CG, 2020. The Covid-19 epidemic [published online ahead of print, 2020 Feb 12]. Trop Med Int Health 25(3). doi:10.1111/tmi.13383.

Wang C, Horby PW, Hayden FG, Gao GF, 2020. A novel coronavirus outbreak of global health concern. Lancet 395:470-3. doi:10.1016/S0140-6736(20)30185-9.

Wang FS, Zhang C, 2020. What to do next to control the 2019nCoV epidemic? Lancet 395:391-3. doi: 10.1016/ S01406736(20)30300-7
Weeberb JR Jr, Koutrakis P, Roig H L, 2015. Spatial distribution of vehicle emission inventories in the Federal District, Brazil. Atmos Environ 112, 32-9.

WHO, 2020. Coronavirus disease (Covid-19). Situation report 46. released on March 6, 2020. Available from: https://www. who.int/docs/default-source/coronaviruse/situationreports/20200306-sitrep-46-Covid-19.pdf?sfvrsn= 96b04adf 4. Accessed:7 March 2020.

Yang Y, Shang W, Rao X, 2020. Facing the Covid-19 outbreak: What should we know and what could we do? [published online ahead of print, 2020 Feb 24]. J Med Virol 92(6). doi:10.1002/jmv.25720

Zhao S, Lin Q, Ran J, Musa SS, Yang G, Wang W, Lou Y, Gao D, Yang L, He D, Wang MH, 2020. Preliminary estimation of the basic reproduction number of novel coronavirus (2019-nCov) in China, from 2019 to 2020: A data-driven analysis in the early phase of the outbreak. Int J Infect Dis 92:214-7. doi:10.1016/j.ijid.2020.01.050.

Zhu N, Zhang D, Wang W, Li X, Yang B, Song J, Zhao X, Huang B, Shi W, Lu R, Niu P, Zhan F, Ma X, Wang D, Xu W, Wu G, Gao GF, Tan W, 2020. A Novel Coronavirus from Patients with Pneumonia in China, 2019. N Engl J Med 382:727-33. doi:10. 1056/NEJMoa2001017. 